\title{
0 ESTADIAMENTO TNM DO CaRCINOMA DE CÉlULAS RENAIS DEVE SER MODIFICADO NOVAMENTE ?
}

\author{
Marcos Francisco Dall'Oglio*, Miguel Srougi, luciano Nesrallah, Kátia M. Leite, Flávio Hering, \\ Alexandre de Campos Bomfim, Adriana Sanudo \\ Trabalho realizado no Departamento de Urologia da Escola Paulista de Medicina- \\ Universidade Federal de São Paulo-São Paulo
}

RESUMO - OBjetivos. 0 comportamento do carcinoma de células renais estádio PTI não está completamente esclarecido. Nós estudamos a presença de fatores prognósticos e tamanho tumoral na recorrência e sobrevida do carcinoma de rim esporádico após tratamento cirúrgico.

Métodos. Foram revisados retrospectivamente 120 pacientes, 93 PTI, nove PT2, II PT3, sete PT4, seguidos após nefrectomia. Foram analisadas sobrevida e recorrência da doença dentro de três grupos de tumores: grupo I: $<4 \mathrm{~cm}$, grupo 2: $4.7 \mathrm{~cm}$ e grupo 3: $>7 \mathrm{~cm}$ e os fatores prognósticos preditivos avaliados foram grau nuclear, invasão microvascular, presença de gânglios comprometidos e degeneração sarcomatosa

Resultados. A freqüência de fatores prognósticos adversos aumenta à medida que aumenta 0 tamanho do tumor. No grupo I tivemos apenas quatro tumores de alto grau e somen- te um apresentou invasão microvascular não havendo gânglios comprometidos ou degeneração sarcomatosa. No grupo 2 havia 16 tumores de alto grau, quatro sarcomatosos, dois com invasão microvascular positiva e dois com gânglios positivos. No grupo 3, encontraram-se 18 tumores de alto grau, 15 com invasão microvascular e sete com gânglios positivos e cinco sarcomatosos. Houve significância estatística na sobrevida câncer específica $(p=0,002)$ e livre de doença $(p=0,0002)$ entre os três grupos.

ConcLusão. A evolução dos tumores PTI é distinta para tumores menores de $4 \mathrm{~cm}$ e de $4.7 \mathrm{~cm}$ cabendo a subdivisão destes dois grupos em Tla e Tlb.

Unitermos: Carcinoma de células renais. Estadiamento. Neoplasias. Neoplasia recorrente. Neoplasias/classificação.

\section{INTRODUÇÃo}

O carcinoma de células renais (CCR), que representa $3 \%$ das neoplasias malignas do adulto e ocupa o terceiro lugar dos tumores genito-urinários em freqüência, vem apresentando aumento de sua incidência de doença localizada nas últimas décadas'. Isto se deve principalmente pelo aumento no número de diagnóstico incidental que era $7 \%$ no início da década de $70^{2}$ para $61 \%$ em $1998^{3}$.

Apesar do estadiamento de Robson ${ }^{4}$ ainda ser utilizado ${ }^{5}$, a expansão do sistema de estádio TNM é resultado de esforços em criar abrangente classificação anatômica para 0 CCR. A aceitação universal deste modelo é extremamente importante porque proporciona uma linguagem comum para tratamento $e$ avaliação prognóstica dos pacientes, bem como pesquisas e publicações. Atualmente estamos no $5^{\circ}$. TNM para carcinoma de célu-

* Correspondência: Rua Manoel da Nóbrega, $n^{\circ} 853$ casa 22 CEP 04001-084-São Paulo-SP-Brasil E-mail:marcosdalloglio@uol.com.br las renais 6 , porém já existem propostas para modificá-lo novamente ${ }^{7,8,9}$.

Estudos atuais relatam excelentes resultados com cirurgia conservadora ${ }^{10}$, no entanto a maioria não compara a sobrevida entre os pacientes com tamanhos tumorais distintos.

Nós, retrospectivamente, avaliamos o tamanho tumoral e a presença de fatores prognósticos na recorrência e sobrevida dos pacientes com CCR com objetivo de rediscutir o ponto de corte do atual TI(TNM- I997), julgando que existe uma diferença substancial no prognóstico dos tumores a partir de $4 \mathrm{~cm}$ de diâmetro.

\section{Métodos}

No período entre janeiro de 1988 e julho de 1999, 128 pacientes com CCR foram operados por um mesmo grupo de cirurgiões nos Hospitais Sírio Libanês e Beneficência Portuguesa de São Paulo sendo avaliados retrospectivamente. A avaliação pré-operatória compreendeu exames hematológicos e exames de imagem como a ultra-sonografia (US), tomografia computadorizada(TC)e/ou ressonância nuclear magnética, urografia excretora, cinti- lografia óssea e radiografia de tórax. O seguimento pós-operatório variou entre 2 e 138 meses (mediano: 33 meses).

Os pacientes foram consultados sobre a participação no estudo através de carta informativa e termo de consentimento pós-informação e posteriormente foi feita análise retrospectiva através dos dados de prontuário. Destes, foram excluídos oito pacientes porque tinham material anatomopatológico insuficiente para revisão ou este era inconclusivo.

As informações clínicas resgatadas destes prontuários incluíram idade, sexo, rim acometido, data do diagnóstico, apresentação clínica inicial, propedêutica, histórias pregressas, antecedentes pessoais e familiares, terapia cirúrgica realizada (radical ou conservadora), laudo anatomopatológico e seguimento pós-operatório.

Todo material anatomopatológico (lâminas coradas com H.E e fragmentos blocados em parafina) foi revisado ao microscópio ótico por um único patologista. 0 acompanhamento pós-operatório foi realizado no consultório e quando a última consulta se deu há mais de três meses houve 
Tabela I - Características demográficas

\begin{tabular}{lc} 
Média de idade + dp & $59,1+12$ \\
Homens & $89(74 \%)$ \\
Mulheres & $31(26 \%)$ \\
Lado D & $60(50 \%)$ \\
LadoE & $56(47 \%)$ \\
Bilateral & $4(3 \%)$ \\
Células claras & 71 \\
Papilar & 28 \\
Cromófobo & 12 \\
Sarcomatoso & 9 \\
Estádiol & $93(77 \%)$ \\
Estádioll & $9(8 \%)$ \\
Estádiolll & $11(9 \%)$ \\
EstádiolV & $7(6 \%)$ \\
\hline
\end{tabular}

confirmação telefônica do estado atual do paciente. Para seguimento pós-operatório foram recomendados os seguintes exames: radiografias de tórax, cintilografia óssea, tomografia computadorizada e/ou ultrasom abdominal, hemograma, cálcio, creatinina, ferritina, antígeno carcinoembrionário, a cada três meses durante o primeiro ano, semestralmente do segundo ao quinto ano e anualmente após este período.

Foi avaliado o diâmetro tumoral com os fatores de mau prognóstico: grau nuclear, invasão microvascular intratumoral, presença de degeneração sarcomatosa e gânglios comprometidos. Também foi verificado recorrência da doença e sobrevida dos pacientes, em três grupos distintos:

Grupo I: tumores menores de $4 \mathrm{~cm}$

Grupo 2: tumores de 4 a $7 \mathrm{~cm}$

Grupo 3: tumores maiores que $7 \mathrm{~cm}$.

Os índices de sobrevida câncer específica e livre de doença foram calculados utilizandose as curvas de Kaplan-Meier e o Log rank test foi utilizado para comparar diferenças na sobrevivência entre os grupos.

\section{Resultados}

Nossa casuística foi composta por 89 homens (74\%) e 31 mulheres (26\%) de 9 a 87 anos $(59,1 \%)$ sendo submetidos a 98 nefrectomias radicais $(81,5 \%)$ e 22 parciais $(18,5 \%)$. $O$ tumor situava-se no rim direito em 60 (50\%), no lado esquerdo em 56 $(47 \%)$ e bilateralmente em quatro (3\%). Um maior número de pacientes teve CCR diagnosticado incidentalmente, foram 69
$(57,5 \%)$ comparados com 5 I tumores sintomáticos $(42,5 \%)$. As características clínicas dos 120 pacientes estudados e o estádio patológico estão listados na Tabela I.

\section{Grupos de pacientes segundo o diâmetro tumoral}

O Grupo I teve um total de 36 (30\%) pacientes com idade média de 60,3 anos (38-76), com seguimento médio de 33,6 meses ( $\mathrm{I}-\mathrm{I} 38 \mathrm{~m})$ e tamanho tumoral médio de 2,6 cm (I,5-3,5cm). O Grupo 2 teve um total de 51 (42,5\%) pacientes com idade média de 58,5 anos ( $9-87$ anos), seguimento médio de 30,9 meses (I-III meses) e tamanho tumoral médio de $5,3 \mathrm{~cm}(4-7$ $\mathrm{cm})$. O Grupo 3 teve um total de 33 pacientes $(27,5 \%)$ com idade média de 60 anos (42-8I anos), seguimento médio de 35,8 meses (2-90 meses) e tamanho tumoral médio de $10,5 \mathrm{~cm}(7,5-19,5 \mathrm{~cm})$.

\section{Características tumorais}

No Grupo I, apenas quatro pacientes (I2\%) apresentavam alto grau histológico (IIle IV); somente um paciente apresentou invasão microvascular (3\%) e não houve presença de gânglios comprometidos.

No Grupo 2, 16 pacientes (32\%) tiveram grau histológico III e IV, I2 (23\%) apresentaram invasão microvascular e dois (4\%) apresentaram gânglios linfáticos, comprometidos.

No Grupo 3, 18 pacientes (55\%) tiveram grau histológico III e IV, I 5 (45\%) apresentaram invasão microvascular, sete (2।\%) apresentaram gânglios positivos.

\section{Tipos celulares}

Houve distribuição homogênea quanto aos tipos celulares, o subtipo células claras, que é o mais comum, foi o predominante nos três grupos, degeneração sarcomatosa só esteve presente em tumores maiores de 4 $\mathrm{cm}$, sendo quatro (8\%) do grupo 2 e cinco (15\%) no grupo 3.

\section{Recorrência da doença}

Ocorreuem média após I6,7 meses (I-5I) da cirurgia sendo evidenciada em 17 pacientes (14\%), dos quais quatro do Grupo 2 e 13 do Grupo 3. Não houve recorrência no grupo I.

\section{Sobrevida}

No total, 10 pacientes (8\%) foram a óbito devido ao carcinoma de células renais. Não houve óbito no grupo I, duas mortes no grupo 2 e oito óbitos no grupo 3 . A sobrevida câncer específica foi 100\%,95\%, 63\% ( $p=0,002)$ e livre de doença $100 \%, 86 \%, 43 \%$ $(p=0,0002)$ para os grupos I, 2 e 3 , respectivamente (Gráficos l e 2).

\section{Discussão}

Nosso trabalho demonstrou que CCR menores de $4 \mathrm{~cm}$ raramente apresentam fatores prognósticos que contribuem com a má evolução. Eles são tumores de baixo grau, raramente apresentam invasão microvascular, não apresentam degeneração sarcomatosa, oferecendo sobrevida câncer específica e livre de doença equivalente a $100 \%$.

O comportamento biológico dos pequenos tumores renais permanece desconhecido ${ }^{11,12}$, mesmo assim a conduta pode ser expectante para casos selecionados ${ }^{13}$, por outro lado, há muito tempo relaciona-se o diâmetro do tumor e potencial maligno ${ }^{14}$.

A incidência de tumores com menos de 4 $\mathrm{cm}$ aumentou de $28 \%$, em 1985, para $61 \%$ em $1995^{12}$ e o tamanho médio dos tumores reduziu de 7,8 cm, em 1989, para 5,3 cm em $1998^{15}$. Quanto à evolução, tumores menores de $4 \mathrm{~cm}$ têm excelente prognóstico quando tratados com nefrectomia radical ou cirurgia conservadora ${ }^{8,10,15}$, contudo resultados semelhantes foram obtidos para tumores inferioresa $7 \mathrm{~cm}$, porém a maioria desta casuística era composta por tumores com menos de $4 \mathrm{~cm}^{16}$. Devido ao tamanho tumoral como um fator prognóstico de doença localizada ainda ser controverso, julgamos a cirurgia conservadora 
Gráfico I - Sobrevida câncer específica. $(p=0,002)$

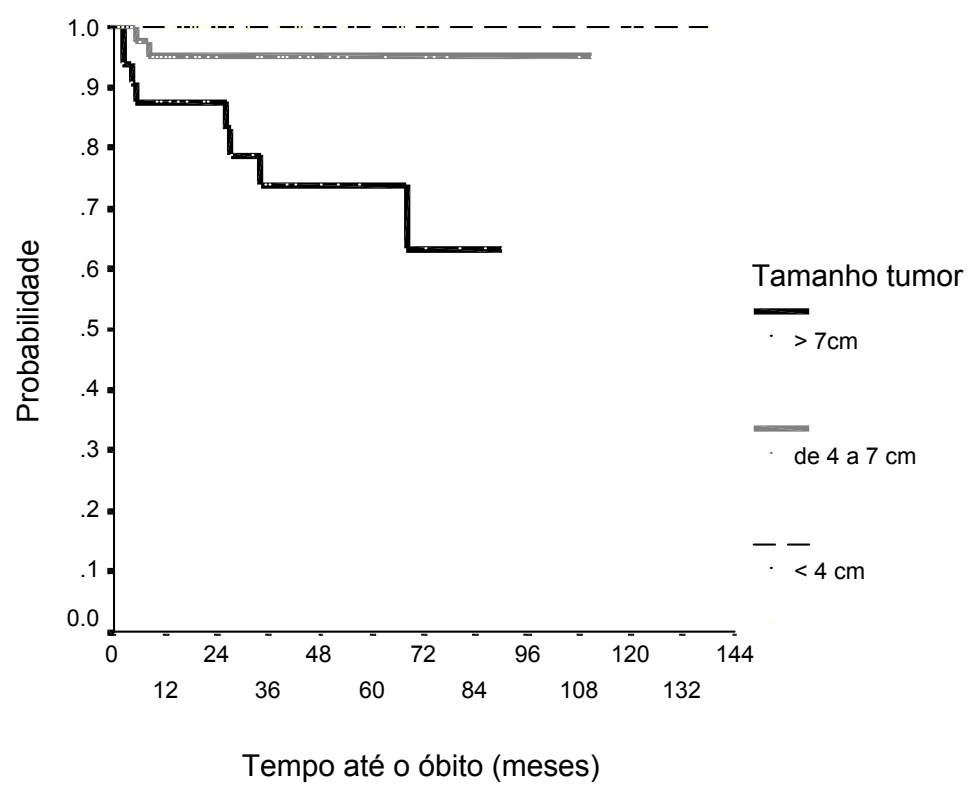

Gráfico 2 - Sobrevida livre de doença. $(p=0,0002)$

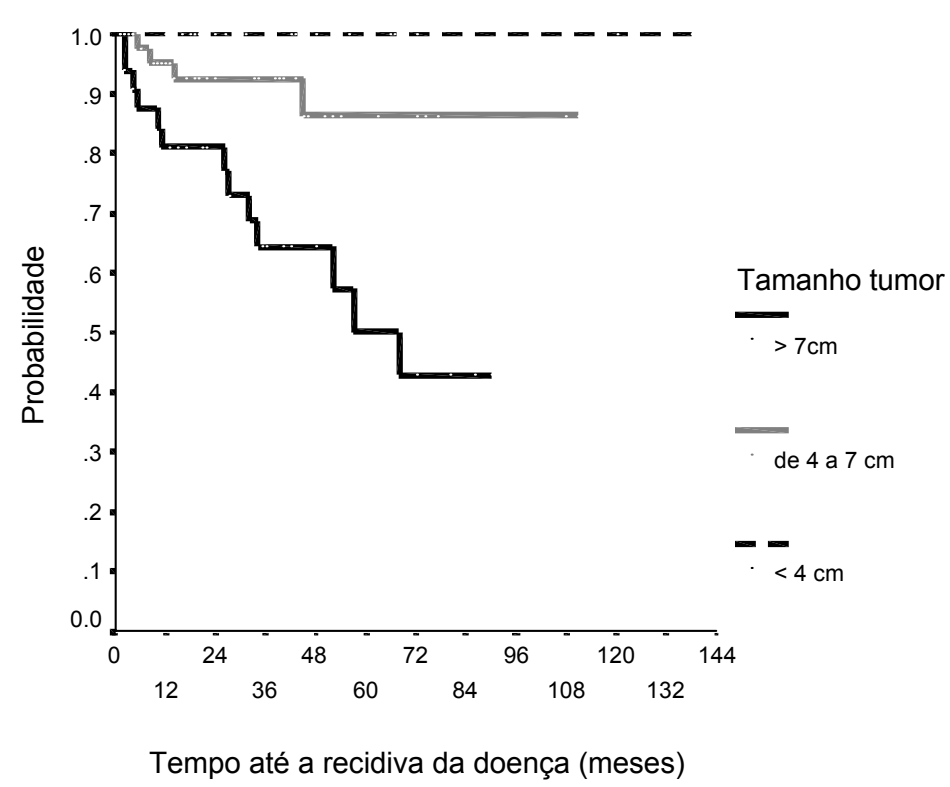

arriscada para tumores de $4-7 \mathrm{~cm}$, pois estes já apresentam fatores de mau prognóstico. Em nossa casuística, no grupo $2(4-7 \mathrm{~cm}) 31 \%$ eram tumores de alto grau, $23 \%$ apresentavam invasão microvascular e $4 \%$ também possuíam linfonodos comprometidos pela doença com $8 \%$ de degeneração sarcomatosa. A cur- va de sobrevida é muito diferente como evidenciam nossos resultados. Existem trabalhos que julgam que o tamanho máximo para realização de cirurgia conservadora é $4 \mathrm{~cm}^{17,18}$.

O estádio da doença é considerado 0 mais importante fator prognóstico em $\mathrm{CCR}^{|9-2|}$. Com o objetivo de universalizar a classificação TNM para CCR, já houve várias mudanças, estando atualmente na quinta edição $0^{6}$. Muitos autores já propuseram modificar o atual estádio TNM com pontos de corte para TI de $4,5 \mathrm{~cm}^{22}, 5 \mathrm{~cm}^{23}$ e $5,5 \mathrm{~cm}^{24}$, justificando a subdivisão em TI para a melhor seleção de candidatos à cirurgia renal conservadora ${ }^{7}$. Javidan et al. ${ }^{25}$ acham que a última classificação TNM (1997) permitirá melhor estratificação de casos de acordo com a sobrevida câncer específica.

Existe uma nova corrente propondo ponto de corte em $4 \mathrm{~cm}$ e a modificação do atual estádio TI (TNM 97) para Tla e TI b7-9 que seria melhor para predizer sobrevida câncer específica e livre de doença otimizando o prognóstico ${ }^{9}$. Nós aderimos a estegrupo, pois pacientes com tumores inferiores a $4 \mathrm{~cm}$ poderiam prescindir do seguimento pós-operatório, se confirmados critérios de bom prognóstico, como jáfoi proposto anteriormente ${ }^{21}$.

Como os tumores menores de $4 \mathrm{~cm}$ raramente apresentam características de mau prognóstico e o estudo do grau nuclear e invasão microvascular exigem análise multivariada, ao passo que o tamanho tumoral já pode ser mensurado antes da cirurgia e tem valor progóstico ${ }^{9}$, o ponto de corte de $4 \mathrm{~cm}$ servirá para otimizar as relações entre tamanho tumoral e os outros caracteres de mau prognóstico. A sobrevida em cinco anos para tumores de alto grau é de $46 \%{ }^{26}$ e gânglios comprometidos equivalente a $33 \%{ }^{2}$, já a presença de invasão microvascular intratumoral confere chance de progressão da doença em

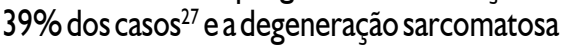
proporciona sobrevida média de 49 meses aos PTI e 6,8 meses aos demais estádios da doen$\mathrm{ç}^{26}$. Como foi evidenciado, os pacientes pertencentes ao grupo I raramente apresentam estes fatores de mau prognóstico. (Tabela 2).

O atual TNM 97 comporta maior número de casos PTI com forte correlação com a sobrevida, pelo fato da doença órgão confinada oferecer evolução satisfatória, com boa distinção prognóstica nos diferentes estádios da doença ${ }^{16,28}$. Como a sobrevida em cinco anos para $\circ$ atual PTI varia entre $85 \%{ }^{29}$ a $91 \%{ }^{26}$, uma das razões de se propor a mudança no TNM seriam as diferenças prognósticas em um mesmo estádio como é evidenciada por Hafez et al. ${ }^{8}$ cuja sobrevida câncer específica para cinco anos foi de $98 \%$, $88 \%$ e $82 \%$ para tumores até $4 \mathrm{~cm}, 4-7 \mathrm{~cm}$ 
Tabela 2 - Características patológicas

\begin{tabular}{cccccccc}
\hline Tumor & $\mathbf{N}^{\circ}(\%)$ & $\begin{array}{c}\text { Tamanho } \\
\text { médio }(\mathrm{cm})\end{array}$ & $\begin{array}{c}\text { Baixo } \\
\text { grau(\%) }\end{array}$ & $\begin{array}{c}\text { Alto } \\
\text { grau(\%) }\end{array}$ & $\begin{array}{c}\text { Invasão } \\
\text { microvascular(\%) }\end{array}$ & $\begin{array}{c}\text { Linfonodos } \\
\text { positivos }(\%)\end{array}$ & $\begin{array}{c}\text { Sarcomatoso } \\
(\%)\end{array}$ \\
$<4 \mathrm{~cm}$ & $36(30)$ & 2,6 & $32(88)$ & $4(12)$ & $1(3)$ & 0 & 0 \\
$4-7 \mathrm{~cm}$ & $51(42,5)$ & 5,3 & $35(68)$ & $16(32)$ & $12(23)$ & $2(4)$ & $4(8)$ \\
$>7 \mathrm{~cm}$ & $33(27,5)$ & 10,5 & $15(45)$ & $18(55)$ & $15(45)$ & $7(21)$ & $5(15)$ \\
\hline
\end{tabular}

e $>7 \mathrm{~cm}$, respectivamente. Nosso trabaIho encontrou sobrevida de 100\%, $95 \%$ e $63 \%$ nos três grupos, respectivamente. Houve clara correlação entre tamanho tumoral e recorrência da doença em nosso estudo. Nossas observações sugerem que tumores com mais de $4 \mathrm{~cm}$ já são potencialmente mais agressivos que tumores menores não devendo compor o mesmo estádio da doença.

\section{ConClusão}

$O$ atual TI do sistema de estádio TNM deve ser modificado em Tla para tumores menores de $4 \mathrm{~cm}$ e TIb para tumores de $4 \mathrm{a}$ $7 \mathrm{~cm}$ por apresentarem características anatomopatológicas e evolução distintas.

\section{SUMMARY}

\section{MUST the TNM StAGING OF THE RENAL CELL CARCINOMA BE MODIFIED AGAIN?}

OBJECTIVE. The behavior of the renal cells carcinoma stage PTI is not completely clarified. We studied the presence of factors after prognostics and tumoral size in the recurrence of survival of the sporadic kidney carcinoma after surgical treatment.

METHODS. 120 patients followed after nephrectomy had been revised retrospectively 93 PTI, 9 PT2, II PT3, 7 PT4, It was analyzed survival and recurrence of the disease inside of three groups of tumors: Group $1:<4 \mathrm{~cm}$, group 2: $4-7 \mathrm{~cm}$ and group 3: $>7 \mathrm{~cm}$, and the prognostics factors above-mentioned evaluated were nuclear degree, microvascular invasion, presence of committed ganglia and sarcomatous degeneration.

RESULTS. The frequency of adverse prognostics factors increase as the tumor size increase. In the group I, we had only four tumors of high degree and only one shown microvascular invasion that does not committed ganglia or sarcomatous degeneration. In group 2 there was 16 tumors of high degree, 4 sarcomatoses, two with positive microvascular invasion and two with positive ganglia. In group 3 , was found 18 tumors of high degree, 15 with microvascular invasion and 7 with positive ganglia and 5 sarcomatoses. There was statistical significance in the specific cancer survival $(p=0.002)$ and free of illness $(p=0.0002)$ between the three groups.

CONCLUSION. The evolution of tumors PTI is distinct for lesser tumors of $4 \mathrm{~cm}$ and 4-7 $\mathrm{cm}$ fitting the subdivision of these two groups in TIa and TIb. [Rev Assoc Med Bras 2003; 49(I): 86-90]

KEYWORDS: Carcinoma renal cell. Neoplasm staging. Neoplasms. Neoplasm recurrence. TNM classification.

\section{REFERÊNCIAS}

I. Chow WH, Devesa SS, Warren JL, Fraumeni JF. Rising incidence of renal cell cancer in the United States. JAMA 1999; 281: 1628-31.

2. Skinner DG, Colvin RB, Vermillion CD, Pfister $R C$, Leadbetter WF. Diagnosis end management of renal cell carcinoma: a clinical and pathologic study of 309 cases. Cancer 1971; 28:1165-77.

3. Jayson M, Sanders H. Increased incidence of serendipitously discovered renal cell carcinoma. Urology I 998; 5 I:203-5.

4. Robson CJ, Churchill BM, Anderson W. The results of radical nephrectomy for renal cell carcinoma. JUrol I 969; 101:297-301.

5. Guinan PD, Vogelzang NJ, Fremgen AM, Chmiel JS, Sylvester JL, Sener SF, et al. Renal cell carcinoma: tumor size, stage and survival: Members of the Cancer Incidence and End Results Committee. J Urol I 995; I 53:90I-3.

6. Sobin, LH, Wittekind CH. International Union Against Center (IUCC). TNM classification of malignant tumors. 5th ed. New York; 1997. p. $180-2$.

7. Guinam P, Sobin LH, Algaba F, Bodellino F, Kameyama S, Mac Lennan G, et al. TNM staging of renal cell carcinoma. American Cancer Society; 1997. p.992-3.

8. Hafez KS, Fergany AF, Novick AC. Nephron sparing surgery for localized renal cell carcinoma: impact of tumor size on patient survival, tumor recurrence and TNM staging.J Urol 1999; 162:1930-3.
9. Igarashi T, Tobe T, Nakatsu HO, Suzuki N, MurakamiS, Hamano M, et al. The impact of a $4 \mathrm{~cm}$. Cutoff point for stratification pf TINOMO renal cell carcinoma after radical nephrectomy. JUrol 200 I; 165: I 103-6.

10. Hafez KS, NovickAC, Butler BP. Management of small solitary unilateral renal cell carcinoma: impact of central versus peripheral tumor location. JUrol 1998; 159: I I 56-60.

II. Nakano E, Iwasaki A, Seguchi T, Kokado Y, YoshiokaY, Sugao H, etal. Incidentally diagnosed renal cell carcinoma. Eur Urol I 992; 2 I:294-8.

12. Wunderlich $H$, Reichelt $O$, Schumann S, Schlichter A, Kosmehl H, Werner W, et al. Nephron sparing surgery for renal cell carcinoma $4 \mathrm{~cm}$. or less in diameter: indicated or under treated? J Urol 1998; I59: I 465-9.

13. Bosniak MA. Observation of small incidentally detected renal masses. Semin Urol Oncol 1995; 13:267-72.

14. Bell ET. Renal diseases. 2nd ed. Philadelphia: Lea \& Febiger; 1950. p.435.

15. Lee CT, Katz J, Shi W, Thaler HT, Reuter VE, Russo P: Surgical management of renal tumors $4 \mathrm{~cm}$. or less in a contemporary cohort. JUrol 2000; 163:730-6.

16. Belldegrun A, Tsui KH, De Kernion JB, Smith RB. Efficacy of nephron-sparing surgery for renal cell carcinoma: analysis based on the new 1997 tumor-node-metastasis staging system.J Clin Oncol I 999; 1 7:2868-75.

17. Licht MR, Novick AC, Goormastic M. Nephron-sparing surgery in incidental versus suspected renal cell carcinoma. J Urol I994; 152:39.

18. Lerner SE, Hawkins CA, Blute ML, Grabner A, Wollan PC, EickholtJT, et al. Disease outcome in patients with low stage renal cell carcinoma treated with nephron-sparing or radical surgery. J Urol 1996; 155: 1868.

19. Russo P. Renal cell carcinoma: presentation, staging and surgical treatment. Semin Oncol 2000; 27:160-76.

20. Gelb AB, Shibuya RB, Weiss LM, Medeiros LJ. Stage I renal cell carcinoma: a clinicopathologic study of 82 cases. Am J Surg Pathol 1993; 17:275-86.

21. Ljunberg B, Alamdari FI, Rasmuson T, Roos G. Follow-up guidelines for nonmetastatic renal cell carcinoma based on the occurrence of metastases after radical nephrectomy. BJU Int 1999; 84:405-II.

22. Zisman A, Pantuck AJ, Chao D, Dorey F, Said JW, Gitlitz BJ, et al. Reevaluation of the 1997 TNM classification for renal cell carcinoma: TI 
Dall'Oglio MF et al.

and $\mathrm{T} 2$ cutoff point at 4.5 rather than $7 \mathrm{~cm}$. better correlates with clinical outcome. JUrol $2001 ; 166: 54-8$.

23. Targonski PV, Frank W, Stuhldreher D, Guinam PD. Value of tumor size in predicting survival from renal cell carcinoma among tumors, nodes and metastases stage I and stage 2 patients. J Urol 1994; I52:1389-92.

24. Kinouchi T, Saiki S, Meguro N, Maeda O, Kuroda M, Usani M, et al. Impact of tumor size on the clinical outcomes of patients with Robson stage I renal cell carcinoma. Cancer 1999; 85:689-95.

25. Javidan J, Stricker HJ, Tamboli P, Peabody JO, Deshpande A, Menon M. Prognostic signi- ficance of the 1997 TNM classification of renal cell carcinoma. JUrol, 1999; |62: |277-8I.

26. Pantuck AJ, Zisman A, Belldegrun AS. The changing natural history of renal cell carcinoma. J Urol 200I; 166:161 I-23.

27. Van Poppel H, Vandendriessche H, Boen K, Mertens V, Goethuys H, Haustermans $\mathrm{K}$, et al. Microscopic vascular invasion is the most relevant prognosticator after radical nephrectomy for clinically non metastatic renal cell carcinoma. J Urol 1997; 158:45-9.

28. Moch H, Gasser T, Amin MB, Torhorst J, Sauter G, Mihatsch J, et al. Prognostic utility of the recently recommended histologic classification and revised TNM staging system of renal cell carcinoma. A Swiss experience with 588 tumors. Cancer 2000; 89:604-I4.

29. Minervini R, Minervini A, Fontana N, Traversi C, Cristofani R. Evaluation of the 1997 tumor, nodes and metastases classification of renal cell carcinoma: experience in 172 patients. BJU Int 2000; 86:199-202.

Artigo recebido: 25/04/2002 Aceito para publicação: 05/07/2002

\title{
À BEIRA DO LEITO
}

Envie sua contribuição para esta nova seção da RAMB, que apresenta perguntas com respostas objetivas sobre condutas práticas. Sua colaboração é muito importante para nós.

\author{
Ramb - Rua São Carlos do Pinhal, 324 - CEP 01333-903 \\ São Paulo - SP - Tel.: (11) 3266-6800 - ramb@amb.org.br
}

